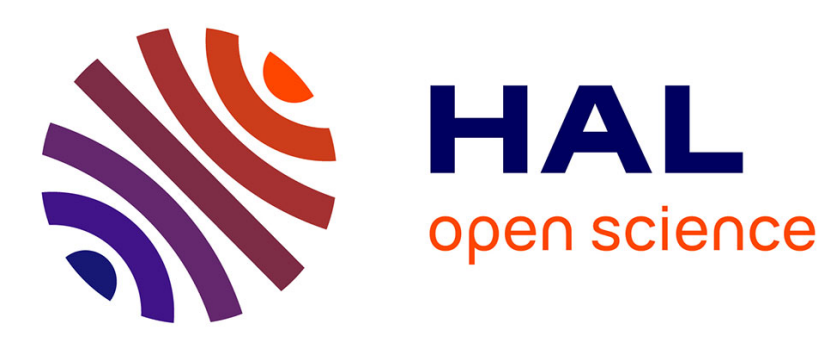

\title{
Patient-specific simulation of guidewire deformation during transcatheter aortic valve implantation
}

Phuoc Vy, Vincent Auffret, Miguel Castro, Pierre Badel, Michel Rochette, Pascal Haigron, Stéphane Avril

\section{> To cite this version:}

Phuoc Vy, Vincent Auffret, Miguel Castro, Pierre Badel, Michel Rochette, et al.. Patient-specific simulation of guidewire deformation during transcatheter aortic valve implantation. International Journal for Numerical Methods in Biomedical Engineering, 2018, 34 (6), pp.e2974. 10.1002/cnm.2974 . hal01833094

HAL Id: hal-01833094

https://hal-univ-rennes1.archives-ouvertes.fr/hal-01833094

Submitted on 13 Jul 2018

HAL is a multi-disciplinary open access archive for the deposit and dissemination of scientific research documents, whether they are published or not. The documents may come from teaching and research institutions in France or abroad, or from public or private research centers.
L'archive ouverte pluridisciplinaire HAL, est destinée au dépôt et à la diffusion de documents scientifiques de niveau recherche, publiés ou non, émanant des établissements d'enseignement et de recherche français ou étrangers, des laboratoires publics ou privés. 


\title{
Patient-specific simulation of guidewire deformation during Transcatheter Aortic Valve Implantation
}

\author{
Phuoc Vy ${ }^{1,2,3,4}$, Vincent Auffret ${ }^{3,4,5}$, Miguel Castro ${ }^{3,4}$, Pierre Badel $^{2}$, Michel Rochette ${ }^{1}$, Pascal \\ Haigron $^{3,4}$, Stéphane Avril ${ }^{2}$ \\ 1 : ANSYS France, 69100 Villeurbanne, France \\ 2: Ecole Nationale Supérieure des Mines de Saint-Etienne, CIS-EMSE, INSERM:U1059, \\ SAINBIOSE, 42023 Saint-Etienne, France \\ 3 : INSERM, U1099, 35000 Rennes, France \\ 4 : LTSI, Université de Rennes 1, 35000 Rennes, France \\ 5 : CHU Rennes, Service de Cardiologie et Maladies Vasculaires, 35000, Rennes
}

\section{Abstract}

Transcatheter Aortic Valve Implantation (TAVI) is a recent mini-invasive procedure to implant an aortic valve prosthesis. Prosthesis positioning in TAVI appears as an important aspect for the success of the intervention. Accordingly, we developed a patient-specific Finite Element framework to predict the insertion of the stiff guidewire, used to position the aortic valve. We simulated the guidewire insertion for 2 patients based on their pre-operative CT scans. The model was designed to primarily predict the position and the angle of the guidewires in the aortic valve and the results were successfully compared to intra-operative images.

The present paper describes extensively the numerical model, which was solved using the ANSYS software with an implicit resolution scheme, as well as the stabilization techniques which were used to overcome numerical instabilities. We performed sensitivity analysis on the properties of the guidewire (curvature angle, curvature radius, stiffness) and the conditions of insertion (insertion force, orientation). We also explored the influence of the model parameters. The accuracy of the model was quantitatively evaluated as the distance and the angle difference between the simulated guidewires and the intra-operative ones.

A good agreement was obtained between the model predictions and intra-operative views available for 2 patient cases. In conclusion, we showed that the shape of the guidewire in the aortic valve was mainly determined by the geometry of the patient's aorta and by the conditions of insertion (insertion force and orientation).

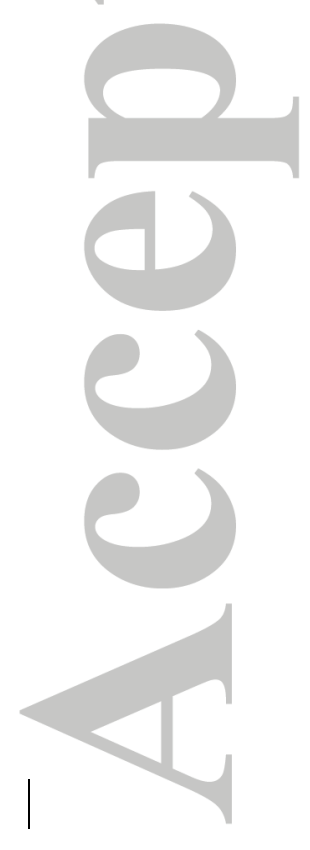




\section{Introduction}

Transcatheter Aortic Valve Implantation (TAVI) is a mini-invasive procedure to replace aortic valves in the context of Aortic Stenosis. This procedure consists in delivering a fully collapsible replacement valve (prosthesis) onto the native aortic valve through a catheter (sheath). A stiff guidewire is first inserted in the patient's aorta with access via the femoral artery. The function of the guidewire is that of a rail along which the sheath can glide. This function is essential to ensure the navigation along the aortic arch up to the heart.

The first TAVI procedure was achieved in 2002 by Alain Cribier [1]. Since then, TAVI has permitted the treatment of patients who cannot benefit of an open heart surgery [2][3]. Nowadays, TAVI shows excellent 1-year outcomes for both high-risk and intermediate-risk patients [4]. Building up on these excellent results, TAVI procedures might drastically increase in the future.

Despite this situation, a number of aspects remain to be improved, including the long term durability and the management of possible complications. Research efforts by medical teams are focused on identifying the mechanisms of complications, and determining the strategies to handle them [5][6]. It was shown that the risk of complications may be increased in case of inappropriate positioning of the delivery system in the valvular plane. For instance, paravalvular leaks [7][8][9], atrioventricular block [10] and coronary occlusion [11] could be related to the invalid implantation position of the prosthesis within the native aortic valve.

Patient-specific numerical simulations can help clinicians during pre-operative planning and intraoperative navigation, as shown by different reviews recently published [12][13][14]. Several studies have already proved the feasibility of simulating prosthesis deployment within patient-specific anatomy. (Bosmans et al., 2016) validated a self-expanding prosthesis deployment model by comparing it with post-operative data. They obtained an accurate prediction of the final shape of the prosthesis [15]. (Wang et al., 2014) simulated balloon-expandable valve deployment within several patient-specific aortic valve geometries [16]. They illustrated how the interaction between calcifications and Valsalva sinuses could possibly induce aortic rupture. Simulation was instrumental in the decision of the medical team for the simulated patient cases. (Auricchio et al., 2014) suggested the measurement of the coaptation area of prosthesis leaflets to estimate the risk of transvalvular leaks [17]. (Morganti et al., 2014) simulated the deformed geometry of deployed prosthesis within the aortic valve [18]. They were able to predict risks of perivalvular leaks by studying the contacts between the stent and the aortic valve leaflet.

Recently, (Morganti et al., 2015) confirmed that the implantation depth and release angle had a crucial role on determining valve anchoring, device deformation and risk of regurgitation [19]. This highlights the importance of correctly positioning the delivery system before and during deployment. However, the simulation of delivery tools (prosthesis, sheath and guidewire) before deployment has not been tackled yet despite their importance on the outcome of the procedure. Such simulation raises many difficult challenges, so we focus on a single tool as a preliminary step towards this goal. To our best knowledge, the stiff guidewire used in TAVI was never simulated, despite the valuable information that can be provided on the mechanical behavior related to tools alignment. To address this issue, we introduce a novel simulation approach of the stiff guidewire in this paper. This novel simulation approach aims at predicting guidewire deformation and positioning in the first stage of TAVI procedures. The paper is organized as follows. In section 2 , the mechanical problem is first formulated from a physical analysis of the stiff guidewire insertion. Then, a numerical model solving this problem is defined. Finally, the methods for our sensitivity analysis and validation are described. The simulation results are reported in section 3 and are discussed in section 4 .

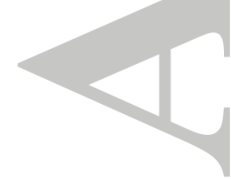




\section{$2 \quad$ Materials and methods}

The purpose of the model is to predict the shape of a stiff guidewire inserted into a patient-specific aortic and ventricular geometry. The mechanical problem consists in determining the stresses and strains of a beam (the guidewire) constrained to fit within a cavity (aorta and left ventricle). The problem is solved with the Finite Element (FE) method using an implicit scheme.

\subsection{Mechanical problem formulation}

\subsubsection{Assumption on motions}

Respiratory and cardiac movements may distort the complete structure [20] [21] [22]. However, the proposed approach was to neglect the dynamic effects of this motion on the guidewire. In addition, guidewire motion was assumed quasi-static because its low mass produced small inertial force compared to the contact forces. Only the final position of the guidewire was predicted by the final static equilibrium of the simulation.

\subsubsection{Mechanical properties}

According to intra-operative observations, stiff guidewire insertion led to marginal deformations of the aorta. Thus, the aortic wall and the ventricle were assumed to remain rigid. Under this assumption, it was not necessary to model the influence of blood pressure, pre-stress within the vascular muscles, and the non-linear behavior of the aortic wall over large deformations. Despite this, a model with linear elastic tissues was tested for the sake of feasibility proof.

Amplatz Super Stiff and Amplatz Extra Stiff guidewires, commonly recommended for the Corevalve $^{\mathrm{TM}}$ and SAPIEN ${ }^{\mathrm{TM}}$ prostheses respectively, were modeled in this study. The elastic properties of the Amplatz Super stiff guidewire used in CoreValve ${ }^{\mathrm{TM}}$ implantation were characterized in prior studies [23][24]. Fig.1.b shows the bending stiffness along the guidewire. We did not observe any permanent deformation on the stiff guidewire caused by the intervention. Excluding gothic aortic arches, we assume that the usual curvature of the aortic arch is not sufficient to cause plastic strains.

The guidewires were designed with a straight end and may perforate the ventricular wall. To minimize the perforation risk, the guidewire has to be curved at its end before the intervention. To that purpose, clinicians manually deform the guidewire to create a curved end, as shown in Fig.1.a. The obtained deformation is permanent (plastic deformation).

This pre-deformation of the guidewire tip was unknown for both patients considered in this study. Therefore, we investigated the impact of this pre-deformation through a sensitivity analysis where we varied both the angle and the radius of curvature. The straight floppy part at the end of the guidewire (Fig.1.a) had a length of $40 \mathrm{~mm}$. The length between the end and point C (Fig.1.a) was set to $120 \mathrm{~mm}$ in agreement to the usual practice in the Rennes University Hospital. This corresponded to the transition between the floppy tip and the stiff body of the guidewire (Fig.1.b).

\subsubsection{Contacts and boundary conditions}

Interactions between the guidewire and the aortic wall result in contact forces. Friction (tangent) contact forces were neglected because the guidewire had a PTFE coating to reduce friction.

In the model, a slipping rail constrained the direction and the orientation of the proximal end (base) of the guidewire in the thoracic aorta. Translations and rotations applied by the operator were modeled as Dirichlet boundary conditions applied at the extreme distal node of the guidewire.

During actual TAVI procedures, the proximal end of the guidewire is free in the left ventricle while the distal end is moved by the operator. Usually, during its introduction through the femoral dilator, 
the curved end of the guidewire can take different orientations, as shown in Fig.1.c and d. Nevertheless, it remains possible to adjust the orientation when the guidewire reaches the ventricular cavity. We defined the model in order to take into account the possibility of different orientations in the ventricular cavity.

\subsubsection{Summary of physical parameters}

The physical parameters input in the model are summarized in Tab.1. They include: the aortic geometry, the guidewire stiffness, the angle and radius of the curved tip, the axial force applied to the distal end of the guidewire and the initial orientation. A sensitivity analysis was performed on these physical parameters to determine how they affect the model predictions. This analysis is designed to provide insights on the behavior of the guidewire rather than provide exact recommendation on the parameters. Indeed, those parameters are difficult to control precisely. The geometry of the stiff guidewire is approximately created as the clinician manually deforms it without any precise tool, or selected in a restricted catalog of pre-shaped guidewire. Likewise, the orientation of the stiff guidewire can be adjusted during its insertion but it is difficult to measure.

\subsection{Discretization \& resolution method}

\subsubsection{Patient specific geometry}

All patients who undergo TAVI at Rennes University Hospital provide informed consent to participate in the French National Transcatheter Aortic Valve Registry, FRANCE-TAVI [25], allowing anonymous collection and processing of their clinical data. These interventions are commonly performed under fluoroscopic control. The $2 \mathrm{D}$ visualization of the aortic root can only be achieved via the injection of a contrast agent. The images used in the present study were retrospectively collected after the procedures of 2 patients who underwent TAVI at Rennes University Hospital. For both, a CoreValve prosthesis (Medtronic ${ }^{\mathrm{TM}}$ ) was implanted from retrograde transfemoral access. These retrospective cases were chosen as both had intra-operative images of sufficient quality to permit their registration to the $3 \mathrm{D}$ pre-operative CT images and to be used for the comparison with numerical simulations. The intra-operative images consisted of digitally subtracted angiography (DSA) acquired during the protocol when the guidewire was inserted in the ventricle of the patient. The ascending thoracic aorta appeared clearly enough in these images thanks to the contrast injection (Fig.2.a).

A 3D pre-operative CT image was routinely acquired for clinical assessment before TAVI interventions. The unsynchronized $\mathrm{CT}$ angioscan acquisition was performed using Discovery CT750HD (General Electric ${ }^{\mathrm{TM}}$ ) (Fig.2.b). A 3D reconstructed image of cardiac structures is shown in Fig.2.c. In addition, the clinical protocol included a synchronized CT-scan showing exclusively the aortic valye. In our study, it was assumed that the ventricle geometry obtained in the unsynchronized CT image was fully dilated and close to diastolic state. This was verified by superimposing the synchronized and unsynchronized CT images.

$3 \mathrm{D}$ reconstruction of the left ventricle and aorta was interactively performed using 3D-slicer [26]. The aortic section was smoothed but did not behave well at the supra-aortic trunks (Fig.2.d). Moreover, the automatic segmentation created an irregular rough surface that is unlike the real aorta or ventricle. The surface was smoothed and imported in ANSYS using Spaceclaim, the integrated CAD module of Ansys. Only the aorta was segmented, leaving aside collateral branches and the supra-aortic trunk. The aorta was segmented from the aortic root to the distal thoracic aorta. The artifacts from the supraaortic trunks were interactively smoothed and a more realistic mesh was recovered (Fig.2.c). This correction must be carefully performed to limit the impact on the results. We assumed that the interactions between the guidewire and the abdominal aorta, the iliac and the femoral artery had a marginal effect on the position of the guidewire at the aortic valve because of the long sheaths specifically used in TAVI procedures. 
This paper mainly focuses on the investigation of patient \#1. The investigation of patient \#2, reported in the Appendix, was aimed at the verification of the applicability of the model on different geometries.

\subsubsection{Meshing}

The geometry was meshed in ANSYS. The rigid aorta was meshed with quadrilateral and triangle contact elements (TARGE170) as shown in Fig.1c. The default mesh had 9610 nodes for patient \#1. The guidewire was meshed with quadratic 3-node beam elements based on Timoshenko beam theory (BEAM189) and line contact elements (CONTA175).

\subsubsection{Resolution scheme}

Neglecting friction and plasticity, the problem was conservative. However, due to contacts and large deflections of the guidewire, it turned to be highly nonlinear. The simulations were performed on the commercial software ANSYS 17.1 with full Newton-Raphson method and sparse matrix direct solver. Time substeps ensuring convergence of the resolution were defined automatically by ANSYS. A bisection - subdivision of a substep - was performed when more than 26 equilibrium iterations were required to converge a substep. The minimum allowed time step was $5.10^{-3} \mathrm{~s}$ for every simulation.

\subsubsection{Boundary conditions and stabilization techniques}

The resolution was achieved in 3 stages, as illustrated in Fig.3. Stage I consisted in deforming the guidewire to match the shape of the centerline of the aorta. Stage II consisted in releasing the kinematic constraints applied at stage I, leading to contact between the guidewire and the aortic wall. In stage III, the end of the guidewire could be moved (axial force or rotation) to simulate the action of an operator.

At stage II, the sudden release of the kinematic constraints is often too abrupt for the Newton Raphson algorithm to face contact nonlinearities. Hence, stabilization techniques were introduced. To this end, springs were connected to the nodes of the guidewire. Longitudinal and torsional springs were compared, with torsional springs turning out to be more successful. For each stabilized node, three torsional springs prevented rotations about $\mathrm{X}, \mathrm{Y}$, and $\mathrm{Z}$ axis. They were defined with COMBIN14 elements. They were attached to guidewire nodes at one end and to a virtual anchoring node (created for that purpose) at the other end. Anchoring nodes had their displacement coupled with that of the respective guidewire nodes. Their rotations, however, were tied to the guidewire rotations with a delay of one loadstep. Torsional springs had high initial stiffness $\left(11.36 \mathrm{~N} \cdot \mathrm{mm} \cdot \mathrm{rad}^{-1}\right)$ so that kinematic constraints could be removed without inducing sudden large displacement. The spring stiffness was decreased at each loadstep by a decay ratio. At the end of the stage II, all torsional springs had a null stiffness.

Two options were considered for the reaction force at the distal end of the guidewire. The first option consisted in adjusting the position of the guidewire during the whole simulation to maintain the desired reaction force. This method will be referred as natural insertion. Conversely, the forced insertion consisted in maintaining the initial depth of insertion throughout stage II.

The distal end of the guidewire was arbitrarily assigned to remain tangential to the centerline of the aorta. It happened that the actual position of guidewires observed intra-operatively at the bottom of the descending thoracic aorta may noticeably deviate from the centerline and be in contact with the wall. However, this was sufficiently far from the aortic root to induce a marginal impact on the results of this study.

Contacts were activated during the stage II. Frictionless contact between patient geometry and stiff guidewire was enforced using an Augmented Lagrangian algorithm. The main parameters determining the behavior of the contact were the contact stiffness coefficient (FKN) and the penetration tolerance (FTOLN). Contact stiffness influenced the contact force applied on the bodies to prevent interpenetration. Nodes with higher penetration than the tolerance do not satisfy the contact 
compatibility which is also a convergence criterion. This means that the solver has to repeat the equilibrium iteration with a higher contact force. Numerous preliminary simulations showed that direct contact between the node at the tip of the guidewire and the ventricle led to divergence of the Newton-Raphson algorithm. This issue was addressed by defining a small rigid sphere at the tip of the guidewire (c.f. Fig.1.a). In general, irregular contact zones such as kinks may lead to large variation of the stiffness matrix of the model which would lead to the failure of the resolution algorithm.

\subsubsection{Summary of numerical parameters}

Numerical parameters are summarized in Tab.1. They include: the mesh density, the number of loadsteps, the initial number of substeps, the predictor (parameter to speed up the process of solving a substep by extrapolating better initial equilibrium iteration), the depth of insertion, the decay ratio of stabilization springs, the contact stiffness and the penetration tolerance.

\subsection{Sensitivity analysis}

\subsubsection{Evaluation criteria}

The success of model predictions was assessed based on the following characteristics: robustness, computation time and accuracy.

\subsubsection{Robustness}

The robustness denoted the ability of the simulation to converge in a mathematically satisfying equilibrium solution regardless of the variation of the input parameters. Convergence or divergence of the solution on a specific range of parameters was a straightforward indicator of robustness. It was observed in preliminary simulations that among all physical parameters, the axial insertion force was the main restriction for robustness (as it induced compression of the guidewire that reached its critical buckling load, which is an instability that cannot be handled with implicit resolution). Thus, maximum converging insertion force was deemed to be a relevant indicator of robustness when varying other parameters and results were presented accordingly.

\subsubsection{Computation time}

The simulations on ANSYS 17.1 were performed on a HP Z800 Workstation having two processors Intel ${ }^{\circledR}$ Xeon ${ }^{\circledR}$ E5620 and 12 GB of RAM. The computation time is reported as the duration of stages I and II of the simulation.

\subsubsection{Accuracy}

We defined the accuracy of the prediction as the degree of similarity between the simulation result and intra-operative data. The primary output of the simulation was the position and angle of the simulated guidewire in the aortic valve plane. The intra-operative image was a $2 \mathrm{D}$ fluoroscopic image, so this comparison was performed by projecting the simulation results according to the current pose of the C-arm. An example of measurement is shown in Fig.4.b, where the projection of the simulation is overlapped with the intra-operative view. The aortic valve plane was manually defined according to the contour of the contrast injection. The distance between the simulation and the data was measured at the aortic valve plane in pixels (pixel size for the intra-operative data used here: $0.308,0.308 \mathrm{~mm}$ at the $\mathrm{C}$-arm detector). The difference of angle was measured in degree.

A 3D/2D registration was necessary to bring the simulation results into the intra-operative coordinate system, as shown in Fig.4.b. It is composed of a transformation representing the C-arm pose, a transformation positioning the preoperative CT-scan in the 3D space and a transformation corresponding to the projection performed during the generation of intraoperative images. Numerous $3 \mathrm{D} / 2 \mathrm{D}$ registration methods can be found in the literature to obtain such transformation [27]. In this 
study, a rigid transform combining 3 translations and 3 rotations was iteratively defined. The $\mathrm{C}$-arm pose and the intrinsic perspective projection parameters were extracted from the DICOM header of the intra-operative data in order to initialize the registration algorithm.

The aortic root of the 2D intra-operative image (Fig.2.a) was manually contoured by an expert. The criterion for registration was the Euclidean 2D distance between the contour of the projected 3D preoperative aorta and the 2D aorta contour observed in the fluoroscopy. A distance map was computed from the $2 \mathrm{D}$ aortic contour with the Chamfer operator. The minimization of the distance criterion was iteratively performed using a Powell optimization algorithm until the estimate of the 3D/2D transform was satisfactory [28]. The resulting projection is shown in Fig.4.a. Two intra-operative angiographies at different pose of the $\mathrm{C}$-arm were available and considered in the registration process for patient 2 .

\subsubsection{Variations of input parameters}

The simulations reported in Tab. 1 are sorted according to the variation of input parameters. Sets \#1-6 are related to the exploration of physical parameters. Sets \#7-12 are related to numerical parameters. The geometry of patient \#1 was thoroughly studied under the 12 simulation sets.

Simulation set \#1 explored the curvature angle of the guidewire tip from $20^{\circ}$ to $120^{\circ}$ with a $20^{\circ}$ step at a fixed radius of curvature of $30 \mathrm{~mm}$. Sets \#2 and 3 explored the radius of curvature from 15 to 30 $\mathrm{mm}$ with a $5 \mathrm{~mm}$ step at a fixed angle of $20^{\circ}$ and $80^{\circ}$ respectively. Set \#4 explored the stiffness of the guidewire, which varied from 20 to $100 \mathrm{GPa}$. Set \#5 explored the orientation from 0 to $180^{\circ}$ with a fixed guidewire shape. Set \#6 tested the forced insertion method to increase the initial depth of insertion. The guidewire was initially $47 \mathrm{~mm}$ deeper when using the forced insertion method. An insertion force of $0.1-0.2 \mathrm{~N}$ as indicated in Tab.1 means that the stiff guidewire was inserted to reach a reaction force of $0.20 \mathrm{~N}$ and then retracted. This allowed comparing the shape of the guidewire at 0.20 $\mathrm{N}$ and $0.10 \mathrm{~N}$. Set \#4 and \#6 simply had the guidewire inserted as much as possible until divergence. Finally, the model with linear elastic tissues was tested for feasibility. The tissues had an elastic modulus of $2 \mathrm{MPa}$ and a Poisson's ratio of 0.49 . Arbitrary boundary conditions were defined to maintain the anatomy. Elastic supports $\left(0.15 \mathrm{~N} \cdot \mathrm{mm}^{-3}\right)$ were defined on the thoracic aorta and the apex of the ventricle. The guidewire had a curvature of $20^{\circ}$ and a radius of $25 \mathrm{~mm}$. It was inserted with a natural insertion method to reach $0.05 \mathrm{~N}$ and $0.1 \mathrm{~N}$. The registration of the simulated deformed aorta was not performed, and accuracy evaluation was not available.

Set \#7 explored the contact stiffness coefficient (FKN) from 0.1 to 10. Set \#8 explored the penetration tolerance (FTOLN) from $0.5 \mathrm{~mm}$ to $4 \mathrm{~mm}$. Set \#9 explored the mesh density of the guidewire (1.4 node $/ \mathrm{mm}, 2.5$ node $/ \mathrm{mm}$ and 5 node $/ \mathrm{mm}$ ). The stiffness of the torsional stabilization springs was adjusted to provide the same initial level of support. Set \#10 explored the decay ratio from 0.75 to 0.9 . In set \#11, the decay ratio was adjusted with the number of loadsteps so that the stabilization springs reached the same stiffness at their complete deactivation across all simulations of the set $\left(10^{-4}\right.$ N.mm.rad $\left.{ }^{-1}\right)$. Thus, the number of loadsteps was explored while keeping the release rate of the stabilization springs consistent. Finally, set \#12 explored the relationship between the number of substep and the predictor.

\section{Results}

From a qualitative point of view, the model was able to converge on a large range of parameters. Tab. 2 reports the computation times, including minimum, maximum and mean values, along with the diverged simulations. Tab. 3 reports the minimum, maximum, standard deviation and mean values of accuracy measurements, i.e., angle difference and distance rounded at the nearest pixel. 


\subsection{Sensitivity to physical parameters}

\subsubsection{Influence of the stiff guidewire properties}

The sets investigating the effect of the curvature angle and radius (\#1, \#2, \#3) converged. The maximum insertion force for convergence of set \#4 is reported in Tab.4b. A linear regression analysis between the maximum insertion force and the stiffness of the guidewire was performed. The regression coefficient is $0.004 \mathrm{~N} / \mathrm{GPa}$ and the coefficient of determination is 0.9984 .

Computation times were similar across those simulations (between $3000 \mathrm{~s}$ and $4000 \mathrm{~s}$ ). The simulated guidewires (\#1, \#2, \#3) projected on intra-operative image are shown in Fig.5 (b, c and d). The standard deviation of the angle difference and distance were low, less than $1^{\circ}$ of angle difference and $3 \mathrm{px}$ of distance. The curvature radius of the guidewire produced the highest standard deviation. The curvature angle produced the lowest standard deviation. The projections of set \#4 were not reported in the figures because the simulated guidewire shapes were too similar.

\subsubsection{Influence of the insertion conditions}

Set \#5 investigating the orientation converged. It required a computation time ranging from $3081 \mathrm{~s}$ to 3982 s. Fig.5.e shows the guidewires of set \#5. The deformation of the tip is noticeably influenced by the orientation of the guidewire. The standard deviation was $2^{\circ}$ and $4.4 \mathrm{px}$ for angle difference and distance respectively. Set \#6 converged when the insertion depth was less than $-18 \mathrm{~mm}$ or included in a range between $-3.9 \mathrm{~mm}$ and $10.8 \mathrm{~mm}$. Further range was not tested. The domain of convergence can be observed in the force versus displacement curve in Fig.6.d. The simulated guidewires at the edge of the domain of convergence are projected in Fig.6 ( $\mathrm{a} b$ and $\mathrm{c}$ ). The variation of insertion depth resulted in a large standard deviation $\left(6.8^{\circ}\right.$ and $\left.12.7 \mathrm{px}\right)$. However, simulated guidewires from forced insertion obtained higher differences with the intra-operative guidewire. The differences produced when varying the insertion force from $0.10 \mathrm{~N}$ to $0.20 \mathrm{~N}$ (natural insertion) for set \#1 are reported in Tab.4.a. The simulated guidewires are projected in Fig.5 ( $a$ and $b$ ). The mean values for the angle difference increased by $3.3^{\circ}$ and distance by 1 px with the increasing force. The standard deviations remained similar for both $0.10 \mathrm{~N}$ and $0.20 \mathrm{~N}$.

\subsection{Sensitivity to numerical parameters}

We observe from Tab. 3 that sets \#7 to \#12 produce very small standard deviation (less than $0.5^{\circ}$ and 0 $\mathrm{px}$ ). We deduce that the variation of the numerical parameters has negligible influence on the accuracy. Instead, they show a strong influence on convergence and computation time. Excessively low contact stiffness resulted in divergence. Using predictor with a large number of substep slowed the simulation and occasionally led to divergence. The decay ratio should be adjusted so that the torsional springs are deactivated with stiffness around $10^{-4} \mathrm{~N} \cdot \mathrm{mm} \cdot \mathrm{rad}^{-1}$. The mesh density had a negligible impact on computation time. Each set is detailed in the following paragraphs.

\subsubsection{Contact conditions}

In set \#7, contact stiffness coefficient of 0.1 diverged. All simulations of set \#8 converged. The physical parameters were angle $=100^{\circ}$, radius $=30 \mathrm{~mm}$, force $=0.22 \mathrm{~N}$ with predictor activated. The standard deviations from both contact stiffness coefficient and penetration tolerance variation were negligible, less than $0.5^{\circ}$ for angle difference and $0 \mathrm{px}$ for distance. 


\subsubsection{Model and solver parameter}

Concerning set \#9, the coarser mesh $(1.4$ node $/ \mathrm{mm})$ had the shortest total computation time (4056 s). The finer mesh (5 node $/ \mathrm{mm}$ ) had the longest computation time (14928 s). Maximum insertion force of the finer mesh was $0.01 \mathrm{~N}$ above that of the coarser mesh. A large fraction of the computation time was spent to compute the last increments of insertion force. The standard deviation was negligible for both angle difference $\left(0.3^{\circ}\right)$ and distance $(0 \mathrm{px})$.

The physical parameters for set $\# 10$ were angle $=20^{\circ}$, radius $=30 \mathrm{~mm}$, force $=0.20 \mathrm{~N}$ with a mesh density of 0.14 node $/ \mathrm{mm}$ and 10 substeps per loadstep. The fastest simulation ran with a decay ratio of 0.87 . During the deactivation of the stabilization springs at loadstep 124, their stiffness was near $10^{-4}$ N.mm.rad ${ }^{-1}$. Lower decay ratio generated instability at each loadstep which resulted in an increased computation time. Higher decay ratio slowed down the removal of stabilization springs. If the spring stiffness was still too high during the loadstep of complete deactivation of stabilization springs, instabilities arose and increased the computation time. The projections of set \#9 appeared undistinguishable one from another. Careful inspection showed that very few pixels were positioned differently. The standard deviation was negligible for both angle difference $\left(0.3^{\circ}\right)$ and distance $(0 \mathrm{px})$. The physical parameters for set $\# 11$ were angle $=20^{\circ}$, radius $=25 \mathrm{~mm}$, force $=0.15 \mathrm{~N}$. The best computation time was $1821 \mathrm{~s}$ (decay ratio $=0.7929,75$ loadsteps). The projections of the simulations appeared undistinguishable except small differences in the ventricle. The standard deviation was negligible for both angle difference $\left(0.2^{\circ}\right)$ and distance $(0 \mathrm{px})$.

Finally, the physical parameters for set $\# 11$ were angle $=20^{\circ}$, radius $=30 \mathrm{~mm}$, force $=0.20 \mathrm{~N}$. A simulation using predictor and 10 substeps per loadstep diverged. The use of predictor and the increase of the substep number did not seem to impact the accuracy of the prediction because the standard deviations were low $\left(0.2^{\circ}\right.$ for angle difference and $0 \mathrm{px}$ for distance). Predictor was beneficial if the number of substeps was set to 1 . Conversely, predictor severely hindered the simulation when the number of substeps was set to 10 .

\section{Discussion}

\subsection{Summary}

This study presented a Finite Element modeling framework for predicting the shape of a stiff guidewire inserted into a patient-specific geometry of the left ventricle and aortic arch. The model was solved with an implicit resolution scheme in ANSYS. Then, the performance of the model was evaluated in terms of accuracy, robustness and speed of resolution. Nonlinearity associated with contacts proved to be a challenging problem for the implicit resolution method. Therefore, stabilization techniques were proposed to enhance the robustness of the model and overcome instabilities. The model was able to explore a wide range of parameters and provide insights on the behavior of the inserted guidewire. The results of the simulations were compared to intra-operative 2D images. Very good agreement could be obtained with respect to the intra-operative guidewire position after a thorough sensitivity analysis of the simulation problem. The guidewire properties which produced the most accurate predictions were the following: curvature of $100^{\circ}$ and curvature radius of $30 \mathrm{~mm}$, insertion force of $0.20 \mathrm{~N}$ with a $90^{\circ}$ orientation.

The main conclusion of the sensitivity analysis was that the patient anatomy itself mostly determined the shape of the guidewire and had a more significant impact than the guidewire curvature properties. This can be explained by the high compliance of the guidewire in its curved part compared to the straight body of the guidewire.

Excluding the patient's geometry, the influence of the physical parameters on the prediction was intricate, and prevented from extracting general trends. This was especially the case for the shape of the guidewire in the left ventricle. Therefore, numerical simulations appeared to be useful to understand the insertion and navigation of endovascular tools in the aortic arch and left ventricle.

\subsection{Contribution and comparison to bibliography}


TAVI interventions heavily rely on the experience of medical teams. Thus, numerical models can be a relevant objective complementary support. Most of the published work was focused on the numerical deployment of the prosthesis regardless of its pre-deployment positioning. To the best of our knowledge, no study was performed on the position of delivery tools and prosthesis at the aortic valve. Yet, it is important to deal with the position of the prosthesis because it is a concern for the success of the deployment.

Similar studies were performed by (Dumenil et al., 2012) (Gindre et al., 2015) for the insertion of guidewires during endovascular aneurysm repair [28][29]. Other studies were dedicated to the insertion of catheter during mini-invasive treatment of cerebrovascular disease [30][31][32][33] (Wang, 1997) (Lenoir, 2006) (Nowinski, 2001) (Cotin, 2015). Among them, (Lawton et al., 2000) described a computationally efficient Finite Element model based on an in-house resolution method [34]. The catheter was modeled as a thin rod and the vessels were assumed rigid like in the present study. (Schafer et al., 2009) presented a method to determine the shape of the guidewire using shortest path algorithms [35].

Each problem was unique and raised different challenges. Cerebrovascular intervention raised challenges by navigating into a complex maze of thin arteries. TAVI and endovascular aneurysm repair raised more concerns on the correct placement of a prosthesis in large vessels. During TAVI, unlike other medical contexts, the guidewire is pushed against the ventricle wall. The force exerted against the guidewire, its curvature and its properties could influence its shape. Beyond the insertion of a stiff guidewire alone, it must be kept in mind that the insertion of the prosthesis and its sheath could also deform the aortic arch.

Models for cerebrovascular treatment navigation usually featured in-house implicit resolution schemes, which seemed to be successful when the vessels were rigid and little stress was generated. Conversely, models for aneurysm repair showed that explicit resolution was successful to predict the large deformations of the tools and the arteries. Likewise, simulation model for the deployment of prosthesis mainly used explicit resolution scheme so far. Despite the widespread use of explicit schemes for similar problems, we successfully used an implicit resolution scheme which is expected to provide more reliable results about stresses, strains and contact forces.

4.3 Interpretation of results

The main findings can be summarized as follows. Concerning the physical parameters, the results showed that the insertion force, orientation and curvature radius strongly impacted the guidewire deformation and position at the plane of aortic valve. The curvature angle and stiffness of the guidewire had a weak influence on the inserted shape. The tests over the numerical parameters showed that they had negligible impact on the accuracy. The base of the guidewire was assumed to have little impact on the accuracy at the aortic valve, so its position was arbitrarily chosen. As such, the base of the simulated guidewire did not match the intra-operative image. The comparison of the simulation in the ventricle was not conclusive. The intra-operative ventricle appeared radiotransparent so the quality of registration could not be assessed in this volume. However, all things considered, the model could reach very good accuracy in predicting the position and angle of the guidewire at the aortic valve.

Throughout the development of the model we observed convergence difficulties for insertion force above approximately $0.20 \mathrm{~N}$ using natural insertion method. A force displacement graph was plotted in Fig.6.d and illustrated the domain where the simulation diverged. An analysis on the guidewire stiffness showed that the maximum insertion force was strongly correlated to the stiffness. However, the guidewire shape at the maximum insertion force was nearly identical whatever the stiffness. Using the forced insertion method, the simulation could overcome the instabilities and reach an insertion force between $0.3 \mathrm{~N}$ and $0.5 \mathrm{~N}$. However, those simulations were even more different from the intraoperative $2 \mathrm{D}$ image, shifting the guidewire at the aortic valve more than $10 \mathrm{px}$. Beyond $0.5 \mathrm{~N}$, the simulation encountered again convergence difficulties.

The insertion of a beam against a wall might be prone to buckling effects, which can create convergence difficulties for an implicit resolution method. Euler's critical buckling load is given by the formula: 
Where $\mathrm{L}$ is the length of the beam, $\mathrm{E}$ is the Young's modulus of the beam (60 GPa), I is the area moment of inertia of the cross section $\left(0.0491 \mathrm{~mm}^{4}\right), \mathrm{K}$ is the column effective length factor wich was assumed to be equal to 2 as we considered one end highly constrained by contact force on the aortic wall (fixed) and the floppy tip of the guidewire free to move. Eq. 1 is consistent with the correlation observed in set \#4 between the stiffness of the guidewire and the maximum insertion force. In order to have a rough evaluation of the critical load for the guidewire, we measured that the length of the guidewire which may be subject to buckling was about $180 \mathrm{~mm}$. Fig. 7 shows the major contact zones on the guidewire and the measurement (in green). We obtained a critical load around $0.22 \mathrm{~N}$ and the proportionality coefficient relating $\mathrm{E}$ to $\mathrm{F}\left(\pi^{2} I /(K L)^{2}\right)$ equal to $0.0037 \mathrm{~N} / \mathrm{GPa}$. This theoretical critical load was in the same range order as the maximum axial forces reached before divergence of our simulations $(\sim 0.2 \mathrm{~N})$. Coincidentally, the theoretical proportionality coefficient was also in the same range as the coefficient deduced from set \#4 (0.0040 N/GPa).

\subsection{Limitations}

One of the main limitations of this study was related to the available data. The pre-operative 3D image was unsynchronized with heart cycle and the valve leaflets could not be extracted. The valve calcifications as seen in Fig.2.b have been neglected and may impact the simulation. They could be added as rigid bodies. However, we observed that the simulated guidewire did not touch the segmented calcifications in those patient cases specifically. The shape of the ventricle was approximate due to heartbeat effects. The creation process of the patient mesh also increased the uncertainties on the geometry of the model. The model required information on the conditions of insertion that could not be obtained from the intra-operative data, such as the inserted length of the guidewire, the insertion force, the orientation and the shape of the curvature of the guidewire. Last, there was a possible mismatch between the model geometry and intra-operative image because of heartbeat and breathing motions. The 2D/3D registration was focused on the ascending aorta, where the contrast due to injection was highest. In the near future, the comparison of the numerical model with intra-operative images of a larger cohort of patients should provide a clearer evaluation of the reliability of the simulations and the validity of the assumptions. Finally, despite the importance of modeling the guidewire deformation, the prosthesis and the sheath should be included into the model in future studies.

The Timoshenko beam formulation was not necessarily the most appropriate choice due to the occurrence of shear-locking in field-inconsistent elements. The effect of shear-locking on accuracy and robustness was mitigated by the use of 3-node quadratic element, but certainly affected the speed of the simulation. A possible direction for future works would be the improvement of the performances by including new element formulations.

\subsection{Application perspectives}

The simulation framework offered interesting insights on the behavior of the guidewire and provided data to improve TAVI simulation outcomes accuracy. The framework is currently not at the stage where it can provide strategies to completely correct misalignment of the prosthesis at the aortic valve but tries to contribute towards this goal.

The prosthesis alignment is greatly determined by the sheath in comparison to the guidewire. The latter still contributes to both the alignment and the stability of the prosthesis. In exceptional cases, this small contribution is enough to allow experienced clinicians improving the alignment of selfexpandable prosthesis by manipulating the guidewire. The insights provided by this simulation framework could be applied to predict whether this kind of maneuver is feasible or beneficial for a given patient anatomy and a given guidewire curvature.

In addition, the handling of the guidewire carries a risk of ventricle damage. The simulation framework could help studying the behavior of the contact between the guidewire and the ventricle to help choosing the guidewire curvature associated with the best stability and the safest distribution of contact forces on the ventricular wall. Currently, there is no general agreement regarding guidewire curvature. Increasingly more clinicians use pre-shaped guidewire $\left(\mathrm{SAFARI}^{\mathrm{TM}}\right.$ ) and some center systematically use the smallest size for simplicity. Some clinicians report that small-sized pre-shape is prone to unwanted migration within the ventricle and provides less stability to the prosthesis 
deployment. The current simulation framework has only been assessed for geometrical parameters at the aortic valve. Future works could be directed towards the improvement of prediction in the ventricle.

Finally, the centerline of the aortic valve is the starting point to visualize the prosthesis deployment. The simulated guidewire is a possible improvement to help the clinicians guess more accurately the alignment of the prosthesis in the pre-operative planning stages and approach more cautiously anatomies which produce severe misalignment and have higher risks of complication. As the model matures, sheath and prosthesis may be included to help the clinician making more accurate predictions. Retrograde trans-femoral access is associated with the highest survival rate, but there could be exceptional patient cases where other accesses provide safer positioning of the prosthesis. Future works could also compare the impact of vascular accesses on prosthesis positioning.

\section{Conclusion}

This paper presented a unique simulation framework to predict the angle and the position of a stiff guidewire in the aortic valve. The accuracy of the model was evaluated with patient intra-operative data and good agreement could be reached. The sensitivity analysis concluded that the shape of the guidewire was mainly determined by the patient geometry and the insertion conditions. The unique use of implicit resolution scheme in this particular context required stabilization techniques to guarantee convergence. However, divergence still occurred when the guidewire was subject to buckling. A larger number of patient cases will be simulated in the future to ascertain the reliability of this method.

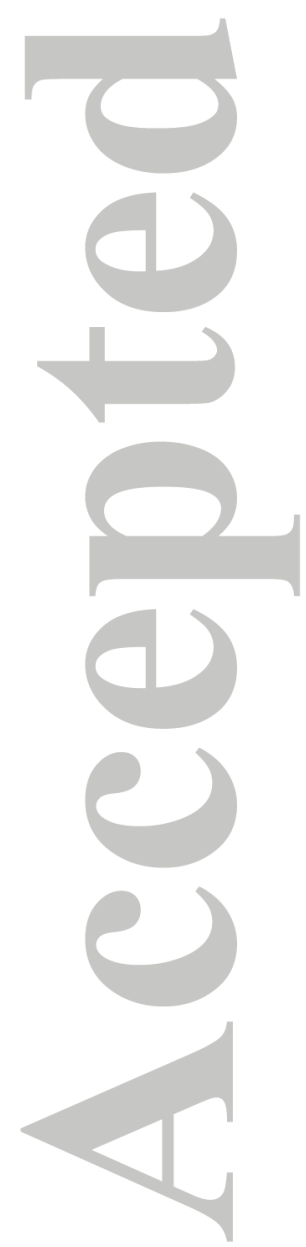


Tab.1 - Variation of parameters organized as sets of simulation, each one focusing on the effect of an individual parameter.

\begin{tabular}{|c|c|c|c|c|c|c|c|}
\hline Physical parameters & $\begin{array}{r}\text { Simulation } \\
1\end{array}$ & 2 & 3 & 4 & 5 & 6 & $7-12$ \\
\hline Focus & angle & radius & radius & stiffness & $\begin{array}{l}\text { orien- } \\
\text { tation }\end{array}$ & $\begin{array}{l}\text { insertion } \\
\text { depth }\end{array}$ & $\begin{array}{l}\text { numerical } \\
\text { param. }\end{array}$ \\
\hline $\begin{array}{l}\text { Guidewire properties } \\
\text { angle }\left(^{\circ}\right) \\
\text { radius }(\mathrm{mm}) \\
\text { base stiffness }(\mathrm{GPa})\end{array}$ & $\begin{array}{r}20-120 \\
30 \\
60 \\
\end{array}$ & $\begin{array}{r}20 \\
15-30 \\
60 \\
\end{array}$ & $\begin{array}{r}80 \\
15-30 \\
60 \\
\end{array}$ & $\begin{array}{r}20 \\
30 \\
\mathbf{2 0 - 1 0 0} \\
\end{array}$ & $\begin{array}{l}20 \\
25 \\
60\end{array}$ & $\begin{array}{l}20 \\
25 \\
60\end{array}$ & $\begin{array}{l}20 \\
30 \\
60 \\
\end{array}$ \\
\hline $\begin{array}{l}\text { Insertion conditions } \\
\qquad \begin{array}{l}\text { force }(\mathrm{N}) \\
\text { orientation }\left(^{\circ}\right) \\
\text { initial depth }(\mathrm{mm})\end{array}\end{array}$ & $\begin{array}{r}0.1-0.2 \\
90 \\
-40\end{array}$ & $\begin{array}{r}0.1- \\
0.2 \\
90 \\
-40\end{array}$ & $\begin{array}{r}0.1-0.2 \\
90 \\
-40\end{array}$ & $\begin{array}{r}\max \\
90 \\
-40\end{array}$ & $\begin{array}{r}0.1-0.2 \\
\mathbf{0 - 1 8 0} \\
-40\end{array}$ & $\begin{array}{r}\max \\
90 \\
7\end{array}$ & $\begin{array}{r}0.2 \\
90 \\
-40\end{array}$ \\
\hline Numerical par & $1-6$ & 7 & 8 & 9 & 10 & 11 & 12 \\
\hline Focus & $\begin{array}{l}\text { physical } \\
\text { param. }\end{array}$ & FKN & FTOLN & mesh & $\begin{array}{l}\text { decay } \\
\text { ratio }\end{array}$ & $\begin{array}{l}\text { decay / } \\
\text { loadstep }\end{array}$ & $\begin{array}{l}\text { substep / } \\
\text { predictor }\end{array}$ \\
\hline $\begin{array}{l}\text { Contact conditions } \\
\text { stiffness coefficient } \\
(\mathrm{N} / \mathrm{mm} 3) \\
\text { penetration tolerance }\end{array}$ & $\begin{array}{l}1 \\
2\end{array}$ & $\begin{array}{r}0.1-10 \\
2\end{array}$ & $\begin{array}{r}1 \\
0.5-4\end{array}$ & $\begin{array}{l}1 \\
2\end{array}$ & $\begin{array}{r}0.1 \\
2\end{array}$ & $\begin{array}{r}0.1 \\
2\end{array}$ & $\begin{array}{l}1 \\
2\end{array}$ \\
\hline $\begin{array}{l}\text { Model \& solver parameters } \\
\text { Mesh element size } \\
\text { Decay ratio } \\
\text { Number of loadsteps } \\
\text { Number of substeps } \\
\text { Predictor }\end{array}$ & $\begin{array}{r}0.4 \\
0.87 \\
125 \\
1 \\
\text { on }\end{array}$ & $\begin{array}{r}0.7 \\
0.87 \\
125 \\
1 \\
\text { off }\end{array}$ & $\begin{array}{r}0.7 \\
0.87 \\
125 \\
1 \\
\text { on }\end{array}$ & $\begin{array}{r}\mathbf{0 . 4 - 0 . 7} \\
0.87 \\
125 \\
1 \\
\text { on }\end{array}$ & $\begin{array}{r}0.7 \\
\mathbf{0 . 7 5 - 0 . 9} \\
125 \\
10 \\
\text { on }\end{array}$ & $\begin{array}{r}0.7 \\
\mathbf{0 . 7 - . 8 7} \\
\mathbf{5 0 - 1 2 5} \\
1 \\
\text { on }\end{array}$ & $\begin{array}{r}0.7 \\
0.87 \\
125 \\
\mathbf{0 1 - 1 0} \\
\text { on/off }\end{array}$ \\
\hline
\end{tabular}


Tab.2 - Computation time of simulations required to reach static equilibrium and remove all stabilization.

\begin{tabular}{|c|c|c|c|c|c|c|}
\hline Set & 1 & & 2 & & 3 & \\
\hline Param. & Angle $\left(^{\circ}\right)$ & $\begin{array}{l}\text { Computation } \\
\text { time (s) }\end{array}$ & \begin{tabular}{|l} 
Radius \\
$(\mathrm{mm})$
\end{tabular} & $\begin{array}{l}\text { Computation } \\
\text { time (s) }\end{array}$ & Radius (mm) & $\begin{array}{l}\text { Computation } \\
\text { time (s) }\end{array}$ \\
\hline $\min$ & 60 & 2772 & 20 & 3045 & 30 & 3289 \\
\hline $\max$ & 120 & 3522 & 30 & 3521 & 25 & 4279 \\
\hline avr & $D$ & 3239 & & 3352 & & 3703 \\
\hline Set & 4 & & 5 & & 6 & \\
\hline Param. & \begin{tabular}{|l} 
Stiffness \\
(GPa)
\end{tabular} & $\begin{array}{l}\text { Computation } \\
\text { time (s) }\end{array}$ & $\begin{array}{l}\text { Orientation } \\
\left({ }^{\circ}\right)\end{array}$ & $\begin{array}{l}\text { Computation } \\
\text { time (s) }\end{array}$ & Depth (mm) & $\begin{array}{l}\text { Computation } \\
\text { time (s) }\end{array}$ \\
\hline $\min$ & 100 & 3435 & 0 & 3081 & -47 & 4638 \\
\hline $\max$ & 20 & 4707 & 180 & 3982 & 7 & 9572 \\
\hline avr & 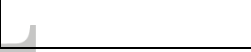 & 3835 & & 3401 & & 7105 \\
\hline Set & 7 & & 8 & & 9 & \\
\hline Param. & \begin{tabular}{|l|} 
Contact \\
stiffness \\
$(\mathrm{N} / \mathrm{mm} 3)$
\end{tabular} & $\begin{array}{l}\text { Computation } \\
\text { time (s) }\end{array}$ & $\begin{array}{l}\text { Penetration } \\
(\mathrm{mm})\end{array}$ & $\begin{array}{l}\text { Computation } \\
\text { time (s) }\end{array}$ & $\begin{array}{l}\text { Mesh } \\
\text { (node/mm) }\end{array}$ & $\begin{array}{l}\text { Computation } \\
\text { time (s) }\end{array}$ \\
\hline $\min$ & 3 & 2846 & 0.5 & 3521 & 1.4 & 4056 \\
\hline $\max$ & 1 & 4113 & 10 & 3702 & 5 & 14928 \\
\hline avr & 1 & 3386 & & 3624 & & 7873 \\
\hline 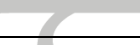 & 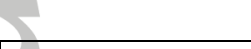 & & & & & \\
\hline Set & 10 & & 11 & & 12 & \\
\hline Param. & Decay & \begin{tabular}{|l|} 
Computation \\
time (s) \\
\end{tabular} & \begin{tabular}{|l|} 
Decay / \\
Loadstep \\
\end{tabular} & \begin{tabular}{|l|} 
Computation \\
time (s) \\
\end{tabular} & \begin{tabular}{|l} 
Substep / \\
Predictor \\
\end{tabular} & $\begin{array}{l}\text { Computation } \\
\text { time (s) }\end{array}$ \\
\hline $\min$ & 0.87 & 31540 & $0.7929 / 75$ & 1821 & $1 / \mathrm{on}$ & 3521 \\
\hline $\max$ & 0.8 & 36623 & $\begin{array}{r}0.8402 / \\
100 \\
\end{array}$ & 2547 & $10 /$ off & 14382 \\
\hline avr & & 33623 & & 2166 & & 7221 \\
\hline Divergec & simulations & & & & & \\
\hline Sets & 12 & & 7 & & 2bis (patient 2 & \\
\hline Param. & Substep & Predictor & FKN & & Angle & Radius \\
\hline Values & 10 & on & 0.1 & & 20 & 20 \\
\hline
\end{tabular}


Tab.3 - Accuracy evaluation of simulation sets from the projection of simulated guidewire on intraoperative image.

\begin{tabular}{|c|c|c|c|c|c|c|}
\hline Set & 1 & angle & 2 & radius & 3 & radius \\
\hline $\begin{array}{l}\text { Parameter } \\
\mathrm{s}\end{array}$ & \begin{tabular}{|l} 
Angle \\
difference $\left({ }^{\circ}\right)$ \\
\end{tabular} & \begin{tabular}{|l} 
Distance \\
$(\mathrm{px})$
\end{tabular} & $\begin{array}{l}\text { Angle } \\
\text { difference }\left({ }^{\circ}\right)\end{array}$ & \begin{tabular}{|l}
$\begin{array}{l}\text { Distance } \\
(\mathrm{px})\end{array}$ \\
\end{tabular} & \begin{tabular}{|l|} 
Angle \\
difference $\left({ }^{\circ}\right)$ \\
\end{tabular} & Distance $(\mathrm{px})$ \\
\hline $\min$ & 3.6 & 2 & -0.5 & 1 & -0.4 & 0 \\
\hline $\max$ & 4.4 & 3 & -2.3 & 6 & -1.6 & 5 \\
\hline mean & 3.9 & 2.3 & -0.8 & 3.3 & -0.7 & 2.3 \\
\hline std & 0.3 & 0.3 & 1.3 & 2.2 & 0.9 & 2.2 \\
\hline Set & 4 & stiffness & 5 & orientation & 6 & depth \\
\hline $\begin{array}{l}\text { Parameter } \\
\mathrm{s}\end{array}$ & $\begin{array}{l}\text { Angle } \\
\text { difference }\left(^{\circ}\right)\end{array}$ & $\begin{array}{l}\text { Distance } \\
(\mathrm{px})\end{array}$ & $\begin{array}{l}\text { Angle } \\
\text { difference }\left(^{\circ}\right)\end{array}$ & $\begin{array}{l}\text { Distance } \\
(\mathrm{px})\end{array}$ & $\begin{array}{l}\text { Angle } \\
\text { difference }\left({ }^{\circ}\right)\end{array}$ & Distance $(p x)$ \\
\hline $\min$ & 0 & 4 & -0.4 & 3 & -0.3 & 0 \\
\hline $\max$ & 0.6 & 5 & 2.8 & 13 & -9.9 & 18 \\
\hline mean & 0.1 & 4.6 & 0.3 & 5.2 & -5.1 & 9 \\
\hline std & 0.3 & 0.5 & 2 & 4.4 & 6.8 & 12.7 \\
\hline Set & 7 & FKN & 8 & FTOLN & 9 & mesh \\
\hline $\begin{array}{l}\text { Parameter } \\
\mathrm{s}\end{array}$ & \begin{tabular}{|l|} 
Angle \\
difference $\left({ }^{\circ}\right)$
\end{tabular} & $\begin{array}{l}\text { Distance } \\
(\mathrm{px})\end{array}$ & $\begin{array}{l}\text { Angle } \\
\text { difference }\left({ }^{\circ}\right)\end{array}$ & \begin{tabular}{|l}
$\begin{array}{l}\text { Distance } \\
(\mathrm{px})\end{array}$ \\
\end{tabular} & \begin{tabular}{|l|}
$\begin{array}{l}\text { Angle } \\
\text { difference }\left({ }^{\circ}\right)\end{array}$ \\
\end{tabular} & Distance $(\mathrm{px})$ \\
\hline $\min$ & 0 & 0 & 0.5 & 2 & 0 & 1 \\
\hline $\max$ & -0.4 & 0 & 1.5 & 2 & -0.5 & 1 \\
\hline mean & -0.3 & 0 & 1 & 2 & -0.2 & 1 \\
\hline std & 0.2 & $\mathbf{0}$ & 0.5 & $\mathbf{0}$ & 0.3 & $\mathbf{0}$ \\
\hline & 10 & decay & 11 & $\begin{array}{l}\text { decay / } \\
\text { loadstep }\end{array}$ & 12 & $\begin{array}{l}\text { substep / } \\
\text { predictor }\end{array}$ \\
\hline $\begin{array}{l}\text { Parameter } \\
\mathrm{s}\end{array}$ & $\begin{array}{l}\text { Angle } \\
\text { difference }\left(^{\circ}\right) \\
\end{array}$ & $\begin{array}{l}\text { Distance } \\
(\mathrm{px})\end{array}$ & $\begin{array}{l}\text { Angle } \\
\text { difference }\left(^{\circ}\right)\end{array}$ & \begin{tabular}{|l} 
Distance \\
$(\mathrm{px})$
\end{tabular} & \begin{tabular}{|l|} 
Angle \\
difference $\left({ }^{\circ}\right)$
\end{tabular} & Distance $(\mathrm{px})$ \\
\hline $\min$ & 1.4 & 4 & 1.7 & 4 & 0.7 & 2 \\
\hline $\max$ & 2.1 & 4 & 2 & 4 & 1 & 2 \\
\hline mean & 1.8 & 4 & 1.9 & 4 & 0.9 & 2 \\
\hline std & 0.3 & $\mathbf{0}$ & 0.2 & $\mathbf{0}$ & 0.2 & 0 \\
\hline
\end{tabular}


Tab. 4 - Detailed measurements for sets \#1 and \#4.

a) Comparison of insertion force $0.10 \mathrm{~N}$ and $0.20 \mathrm{~N}$ (set \#1).

b) Maximum force insertion appears linearly dependent on stiffness of the guidewire (set \#4).

a)

\begin{tabular}{|c|c|c|c|c|c|}
\hline \multirow{2}{*}{\begin{tabular}{|l|} 
Set \#1 \\
Angle $\left(^{\circ}\right)$ \\
\end{tabular}} & & \multicolumn{2}{|c|}{ Insertion force $0.10 \mathrm{~N}$} & \multicolumn{2}{|c|}{ Insertion force $0.20 \mathrm{~N}$} \\
\hline & & $\begin{array}{l}\text { Angle difference } \\
\left({ }^{\circ}\right)\end{array}$ & $\begin{array}{l}\text { Distance } \\
(\mathrm{px})\end{array}$ & $\begin{array}{l}\text { Angle difference } \\
\left({ }^{\circ}\right)\end{array}$ & $\begin{array}{l}\text { Distance } \\
(\mathrm{px})\end{array}$ \\
\hline & 20 & 3.6 & 3 & 0.9 & 2 \\
\hline & 40 & 3.6 & 2 & 1 & 1 \\
\hline & 60 & 3.8 & 2 & 0.9 & 1 \\
\hline 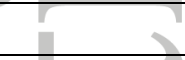 & 80 & 4 & 3 & 0.5 & 0 \\
\hline & 100 & 3.9 & 2 & 0 & 1 \\
\hline 8 & 120 & 4.4 & 2 & 0.2 & 1 \\
\hline Mean & & 3.9 & 2.3 & 0.6 & 1.0 \\
\hline $\begin{array}{l}\text { Standard } \\
\text { deviation }\end{array}$ & & 0.3 & 0.5 & 0.4 & 0.6 \\
\hline
\end{tabular}

b)

\begin{tabular}{|l|r|r|r|}
\hline \multicolumn{5}{|l|}{ Set\#4 } & \multicolumn{3}{|c|}{} \\
\hline Stiffness (GPa) & Angle difference $\left(^{\circ}\right)$ & Distance $(\mathrm{px})$ & Maximum force (N) \\
\hline 20 & 0.1 & 5 & $\mathbf{0 . 0 8}$ \\
\hline 40 & 0 & 5 & $\mathbf{0 . 1 6}$ \\
\hline 60 & -0.2 & 5 & $\mathbf{0 . 2 4}$ \\
\hline 80 & 0.6 & 4 & $\mathbf{0 . 3 2}$ \\
\hline 100 & 0.1 & 4 & $\mathbf{0 . 3 9}$ \\
\hline Mean & 0.1 & 4.6 & \\
\hline Standard deviation & 0.3 & 0.5 & \\
\hline
\end{tabular}


Fig.1

a) Model of guidewire pre-deformation; A: angle; R: radius of curvature; C: start of the curved shape; The tip has a rigid sphere to avoid convergence issues arising from contacts (c.f. section 3-d).

b) Stiffness profile along the guidewire based on (Luboz et al., 2011)(Harrison et al., 2011).

c) d) Definition of orientation of the tip of the guidewire.

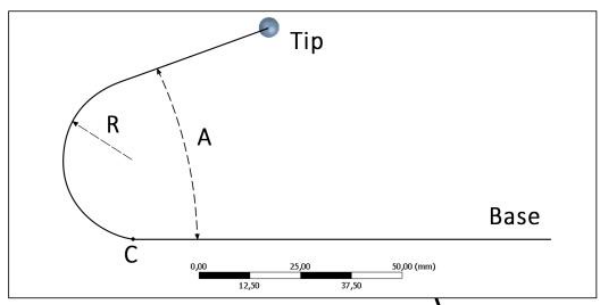

c)

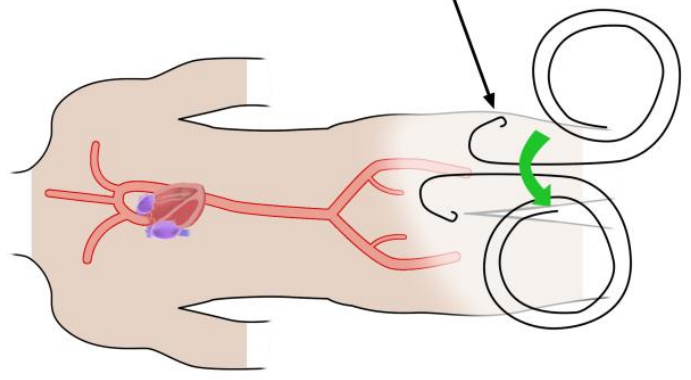

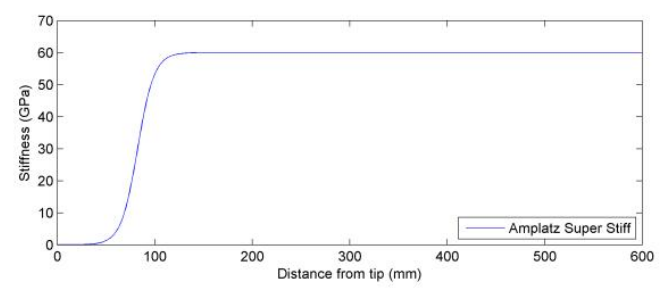

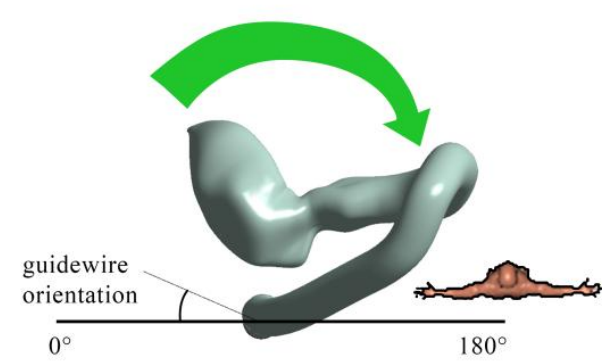

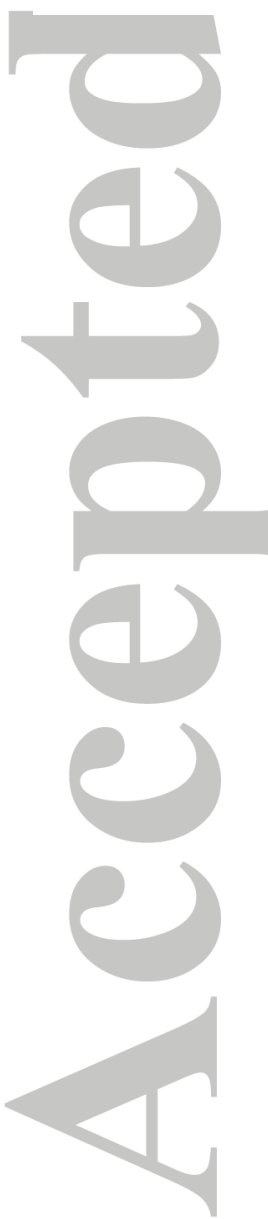


Fig.2

a) Intra-operative angiography of aortic root.

b) Reformatted slice showing the aortic valve from pre-operative CT-scan.

c) Finite Element mesh from segmentation of CT-scan

d) Segmentation from CT-scan

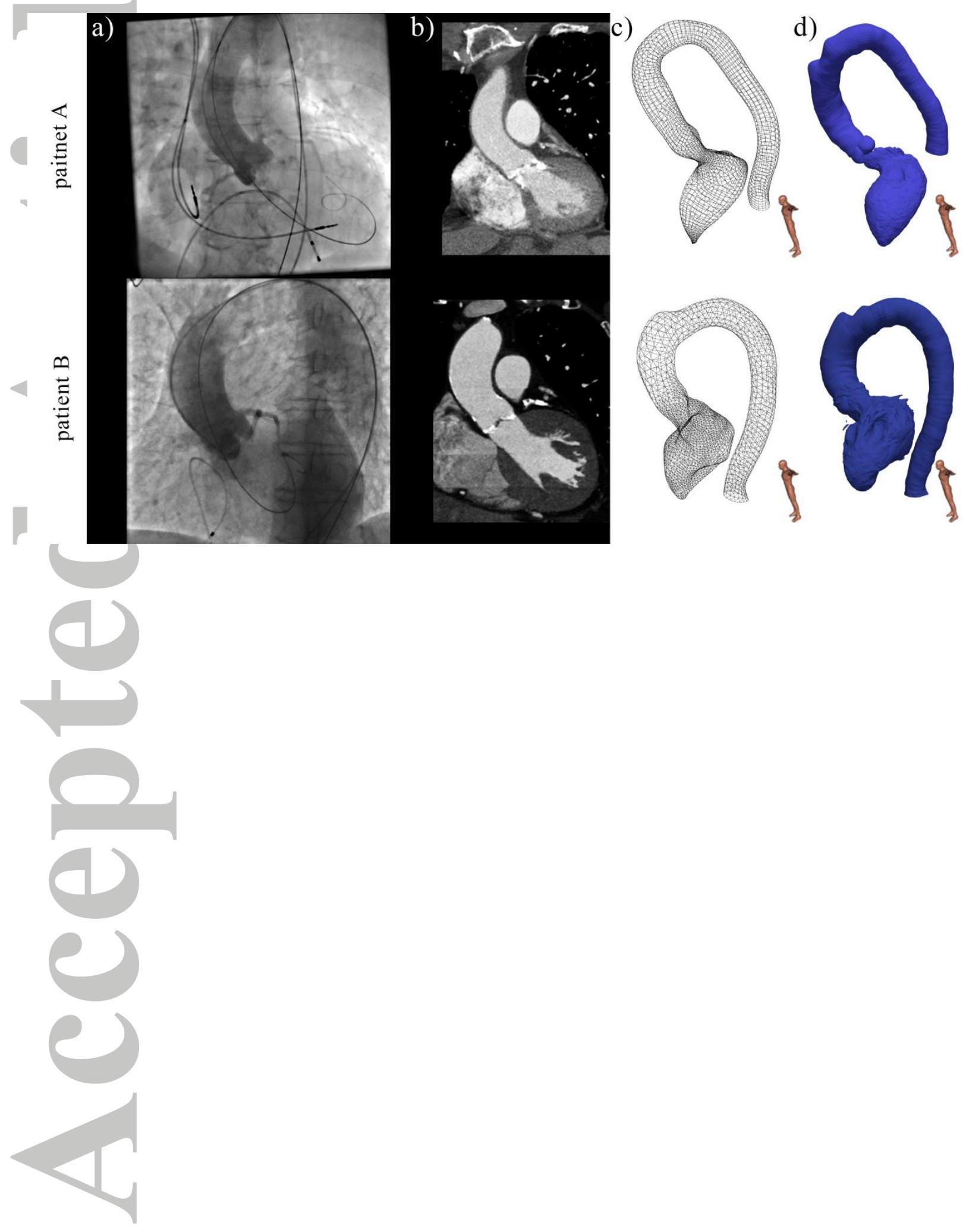


Fig.3 - Illustration of the simulation stages. After applying initial conditions, the guidewire was constrained on the centerline. After the constraints were relaxed, the guidewire reached its final equilibrium. Then insertion force could be varied.

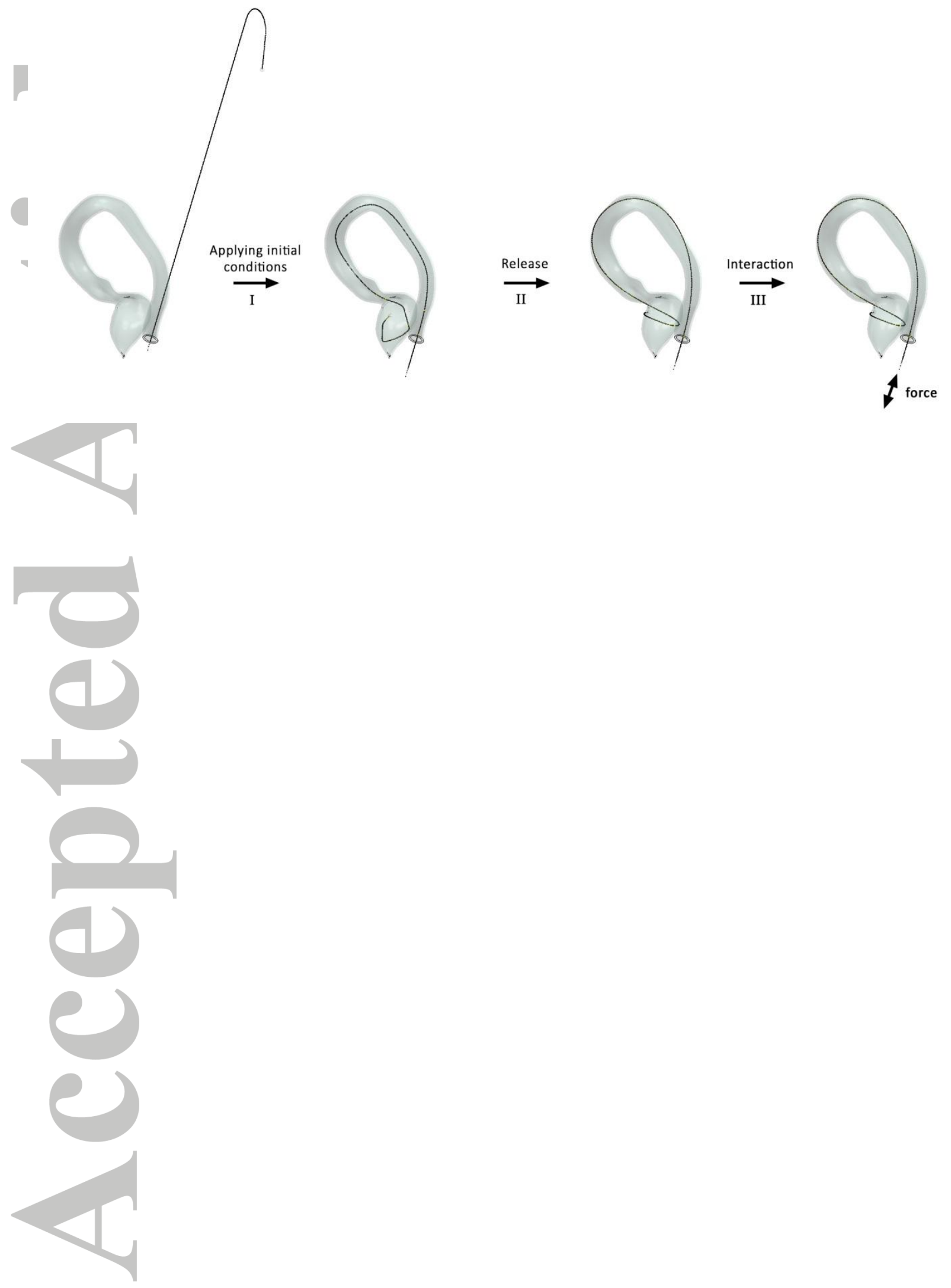


Fig.4

a) Projection of $3 \mathrm{D}$ pre-operative geometry on the intra-operative $2 \mathrm{D}$ view according to the registration transforms.

b) Intra-operative guidewire is drawn with a yellow line; simulated guidewire appear with a cyan line. The aortic valve plane is manually drawn. The distance $\mathrm{D}$ and angle $\mathrm{A}$ of the guidewires are measured at the intersection with the valve plane.
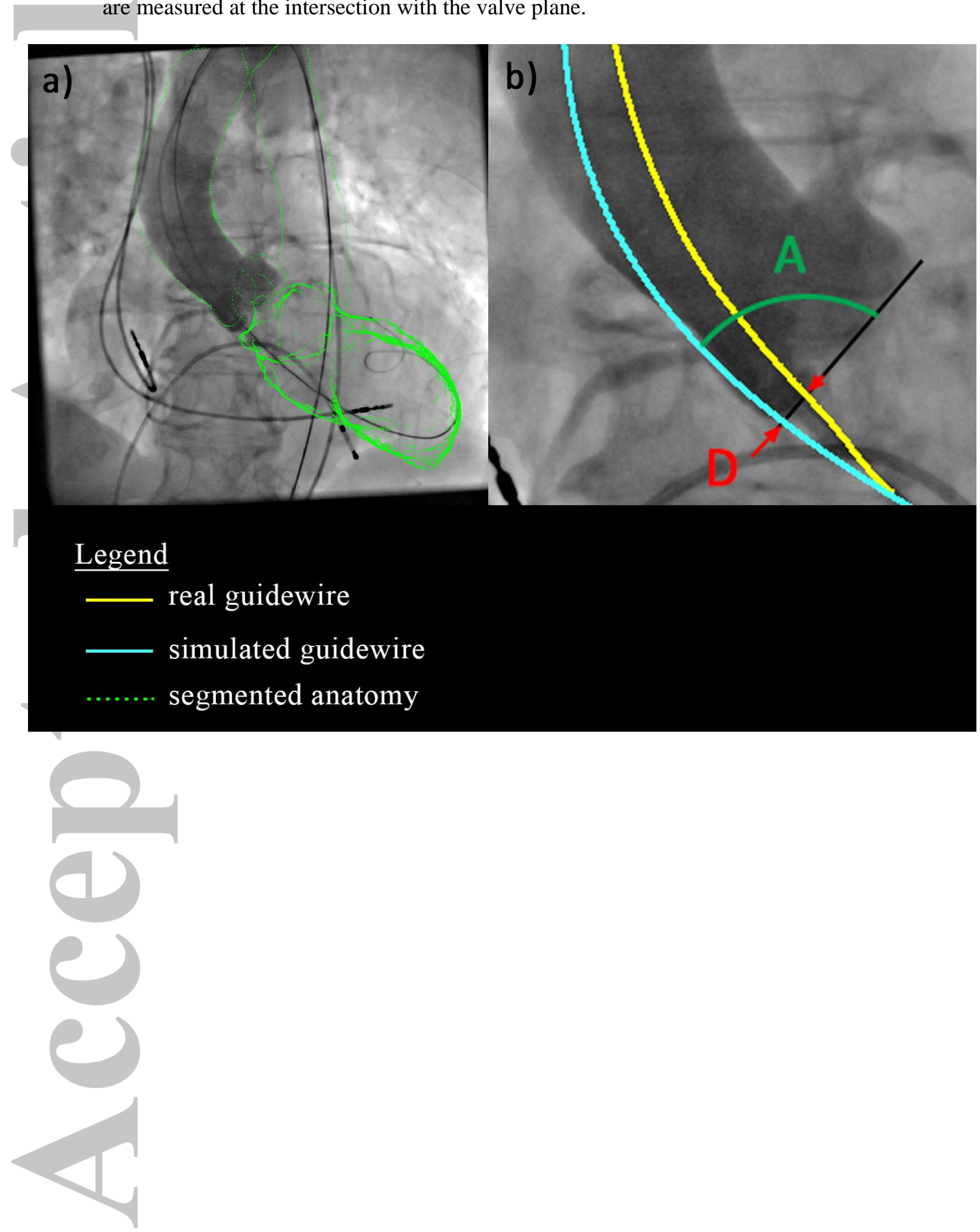
Fig.5 - Projection of simulated guidewires.

a) Set \#1 exploring angle curvature at $0.10 \mathrm{~N}$ insertion force.

b) Set \#1 exploring angle curvature at $0.20 \mathrm{~N}$ insertion force.

c) Set $\# 2$ exploring radius at $20^{\circ}$ angle curvature.

d) Set \#3 exploring radius at $80^{\circ}$ angle curvature.

e) Set \#5 exploring orientation

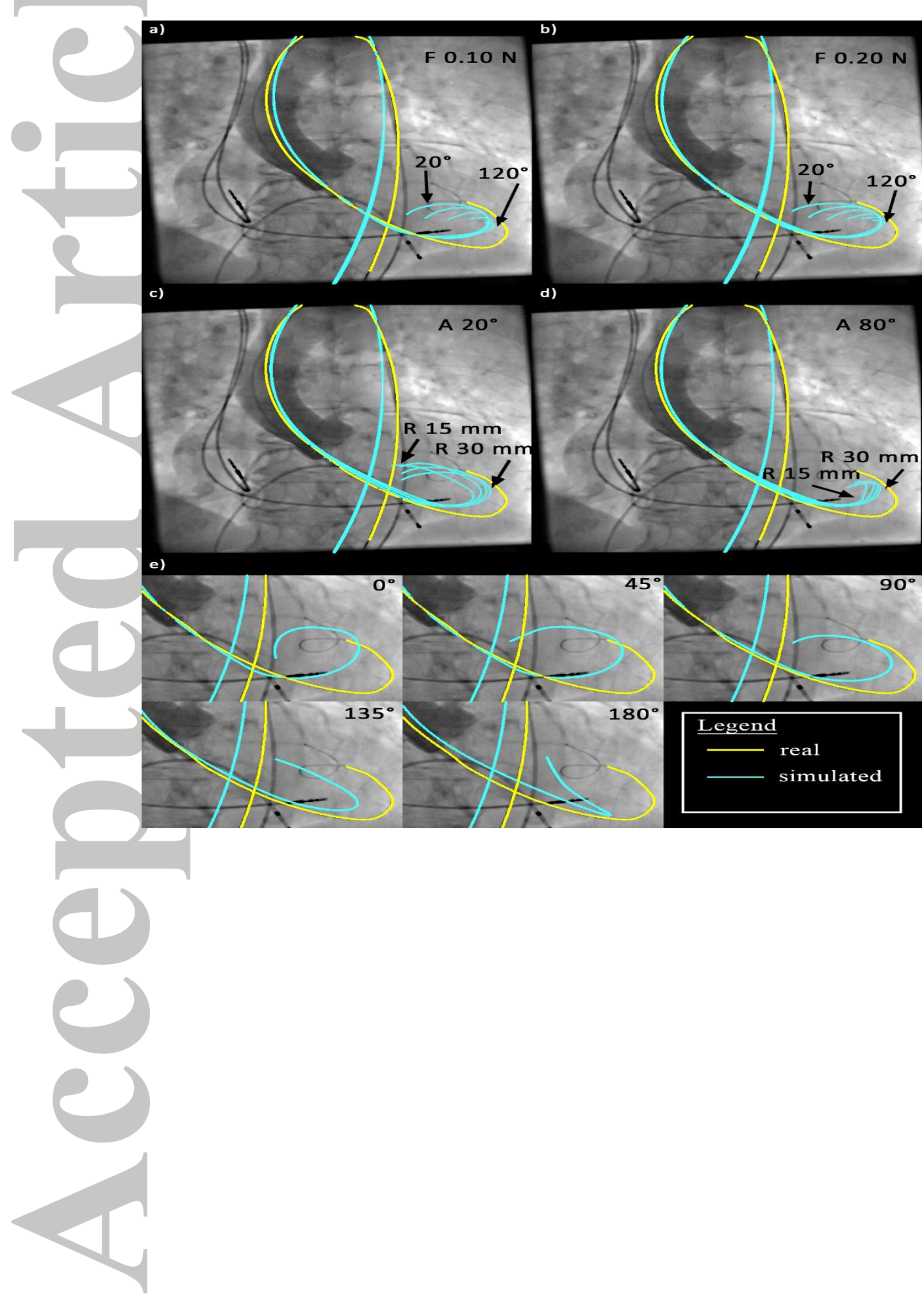


Fig.6

a) b) c) Projections of simulated guidewires at the edges of the convergence domain reported in d). Natural insertion simulations matched closer to intra-operative data than forced insertion simulations. d) Force-displacement graph of the insertion of guidewire. Forced insertion method allows exploring isolated domain of convergence.

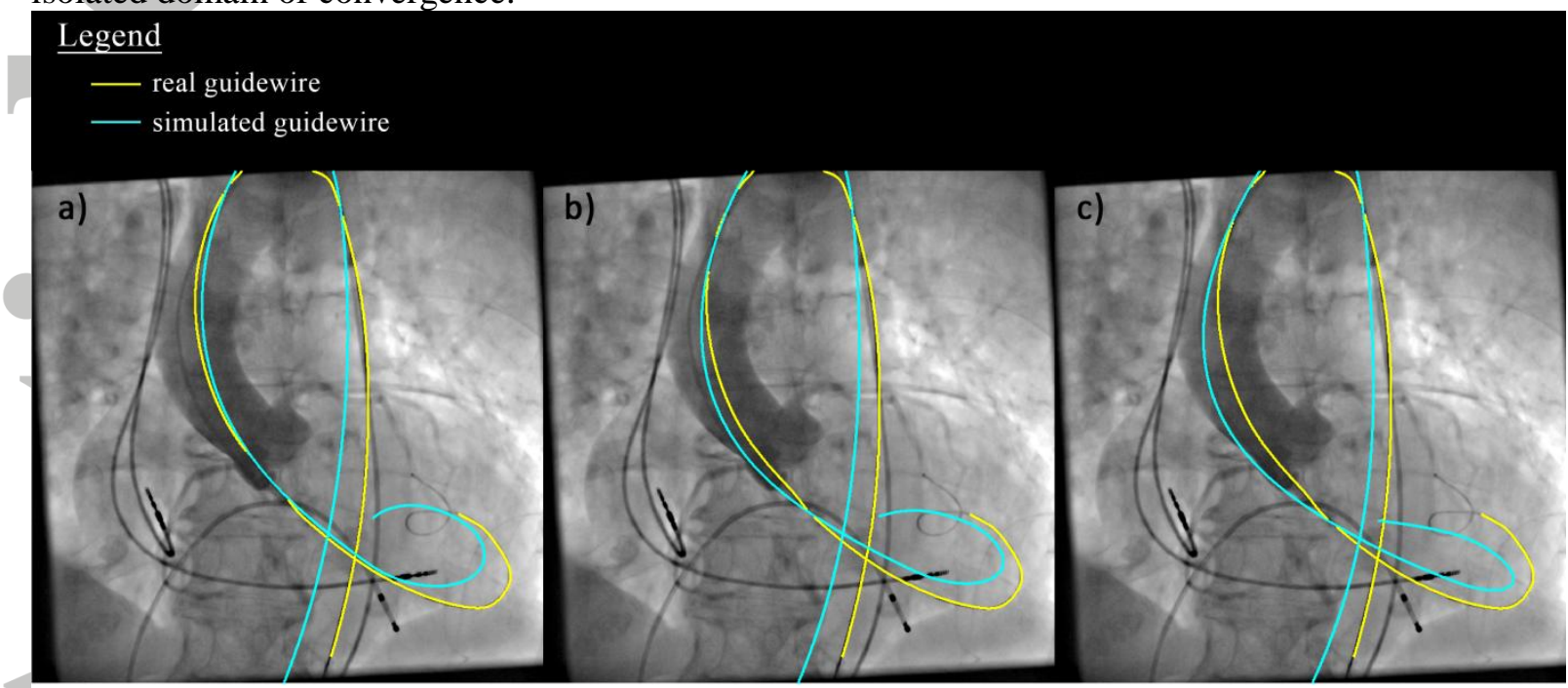

d)
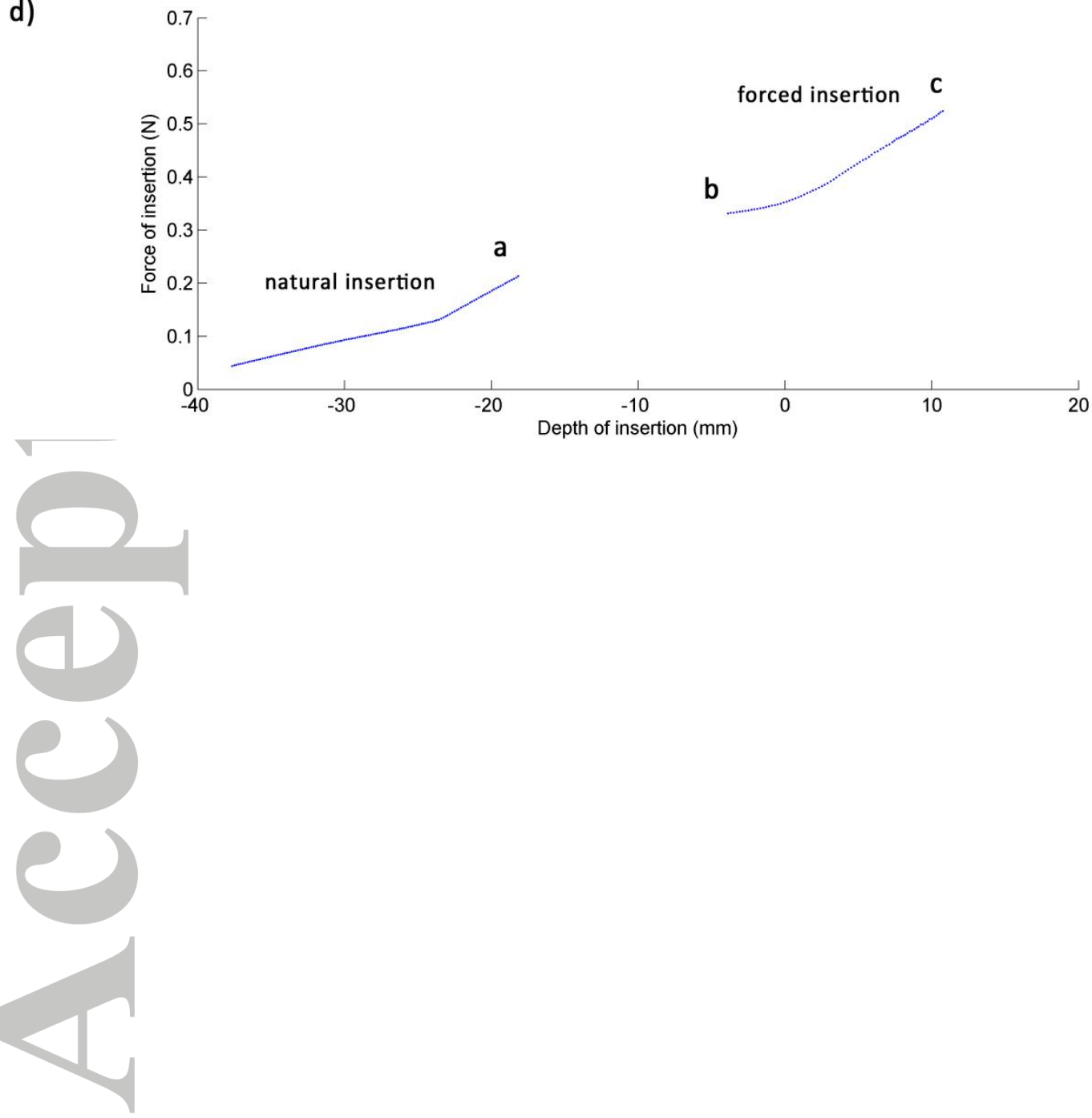
Fig.7

a) Contact force distribution along the simulated guidewire from set \#6 (fig.6a). The green circles highlight contact zones which may produce a buckling effect.

b) Measurement of the portion of the guidewire hypothetically subjected to buckling.

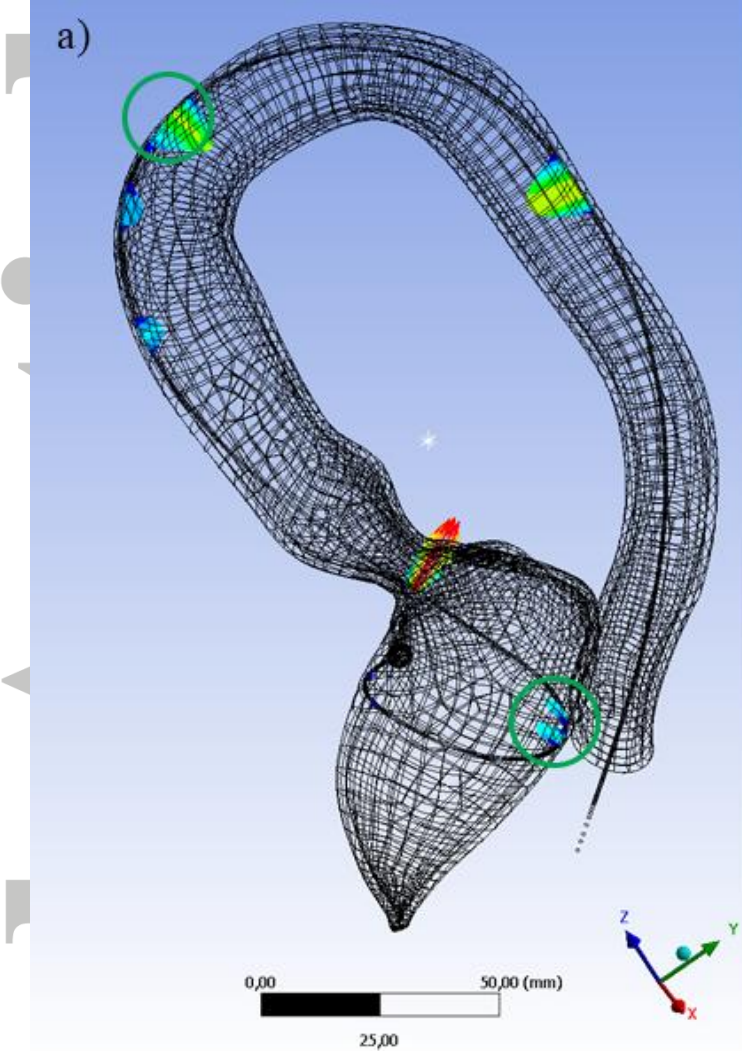

\section{Legend}

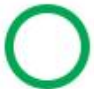

Contact zones possibly related to buckling

Nodal contact force (colour related to intensity distribution) b)

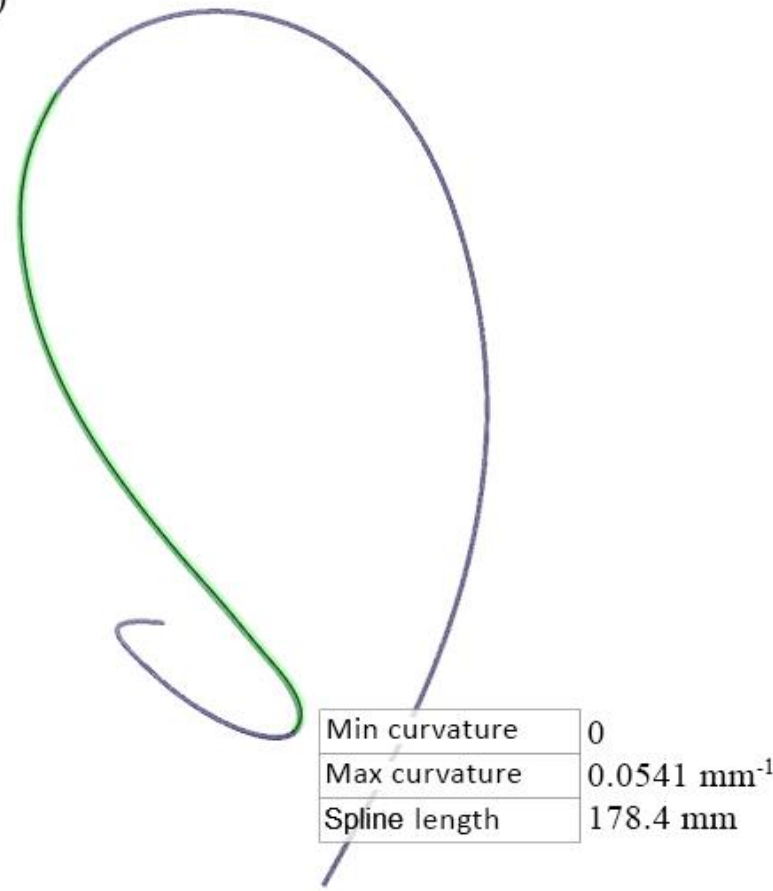

Portion of the beam subject to buckling 